Review article

\title{
Slippery slopes in public health practice
}

\author{
Mathew George \\ Centre for Public Health School of Health Systems Studies Tata Institute of Social Sciences, Deonar, Mumbai, 400088, India
}

\section{A R T I C L E I N F O}

\section{Keywords:}

Public health practice

Susceptible population

Public health evidence

\begin{abstract}
A B S T R A C T
There has been an increased focus to the academic field of public health in India in recent decades. There exist ambiguities and misunderstandings about this domain of expertise both in terms of its field of engagement of problem identification, namely, population, and in terms of its proposed solutions, namely, public health programmes. Public health professionals need to be sensitive to the social context in which the science is practiced and incorporate it into the principles of the profession, which is necessary for improving population health, which in turn help raise the knowledge in the discipline to newer heights.
\end{abstract}

The field of public health as a profession has come to the limelight more than ever during the recent COVID 19 pandemic across the world. It is a practicing profession with a rich history and tradition of successfully intervening to prevent epidemics and infections both in developing and developed nations. The inherently interdisciplinary nature grounded within diverse societal contexts along with a strong people centric approaches applied through its tools like epidemiology has all contributed to the emergence of public health as a strong academic discipline. There has been an increased focus to the academic field of public health in India too in the last two decades. ${ }^{1}$ Despite these, there exist ambiguities and misunderstandings about this domain of expertise due to its inevitable close engagement with the medical profession and the latter's dominance within any country's health services. ${ }^{2}$ In Indian context, the vertical disease control programmes that was implemented in the country during 1950s and 60s and has continued till recent times with or without international funding has been perceived by some as the 'face' of public health programmes. This include the most popular family planning programmes, immunization programme and other disease control programmes, which, is also an important topic covered in the public health curriculum across institutions.

\section{Population is not just an aggregate of discrete individuals}

Two interpretations of 'public' in public health becomes crucial not only in terms of understanding the profession better but also in analyzing the government policies under the rubric of public health as this will help alleviate some of the misconceptions surrounding the field. The first is that public implies population and implies collective interest over individual concerns in its approach. Its corollary is that it is wrong to interpret public as discrete individuals within a society. The latter interpretation possibly emerges from the misinterpretation of public health as a sub discipline of medicine wherein for medical practice every individual is treated as discrete entities. This understanding is also dominated in the scientific epidemiology. ${ }^{3}$ Historical evidence reveals that population level interventions were more successful, effective, and sustainable than individual level approaches for addressing public health problems. ${ }^{4}$ Here, the idea of population in public health owes its understanding to the field of social sciences and is based on holism where the whole is always more than the sum of its parts. Intervening at a population level effectively calls for the need to understand the societal context of population, in terms of the social groups and inequalities and the structure and questions of power. The practical implications of this understanding are that while dealing with any epidemic, it is important to consider the diverse social, economic, cultural and other characteristics of people and their lived experiences in a society. This call for the need to collect diverse set of information pertaining to social groups for better analysis thus ensuring the need for better data collection during epidemics, a basic prerequisite for any meaningful social analysis. What this translates to is that during epidemics and in interpreting any public health problems, the information related to the occupation characteristics, caste, race and socioeconomic status along with rural and urban differentials are equally or more important than the variables like age and sex related information, the former categories are ignored in the context of current epidemic. COVID 19 related analysis across states in India has not yet focused on the range of occupation groups and caste groups and their distribution possibly due to the absence of data elements.

Another important dimension of population that is crucial in public

E-mail address: mathewg@tiss.edu. 
health is the 'scale' in which it is used in the context of epidemics. What is an ideal way to interpret the size of a population (denominator) in the context of an epidemic? It is surely not based on the administrative boundaries of the state or district as microbes don't spread according to state or national boundaries. In traditional epidemiology, the population that is susceptible to the epidemic in a specific duration and is understood as a function of the health behavior of the people, infectivity of the disease, its mode of spread and so on. ${ }^{5}$ This must be realistically examined based on population level studies instead of merely assuming the population within an administrative boundary as equally susceptible, one of the assumptions usually made in modelling and projections based on convenience. This was obvious when cases were confined in few cities during the early phase of the pandemic, when the total population of the country or the state was taken as susceptible population in several of the estimations and projections, which was later proved wrong. ${ }^{6}$ Adding to this is the challenging question about the diverse contextual factors across population that decide high or low susceptibility across social groups. A more realistic and accurate interpretation of susceptible population might call for the need to have a better contextual understanding of the population. It is true that smaller community characteristics help understand and interpret the disease dynamics and its nature of spread better and can create possible ways to prevent the spread than those covering a larger scale. Whatever success stories that have reported across the country during early phases of COVID 19 even for shorter durations were all specific small-scale interventions within specific contexts. ${ }^{7,8}$ The question on the ideal size of population is also applicable when aiming at herd immunity and disease control and so on. For instance, What is the ideal size of the population for which herd immunity can be achieved? Can we even imagine achieving herd immunity for a block, district, or a state or only applicable for entire nation or regions? Historically, it was the contextual, micro level interventions that had successfully reduced the spread of epidemics that qualify first as a public health evidence, which then got upscaled to a larger population with necessary modifications and thus developed as a successful public health programmes nationally and even globally. What is currently missing is these successful micro-level interventions, which has to be a prerequisite for any large-scale programme to claim itself as a public health programme.

\section{Public health evidence: a pre-requisite for public health programmes}

The second and the more challenging misconception leading to slippery slope in public health practice is when 'public' in public health is understood as government as in the case of public policy. It is advisable and suggestive for the national governments to take dominant role in public health practice. There also exist a range of civil society agencies and other private initiatives across the country implementing public health programmes with or without the support of the government. The implication is that all the activities government does in the field of health by default is labelled as public health, irrespective of its capacity to prevent the occurrence of health problems, the primary mandate of public health programmes. Adding to this is the ambiguity existing in a mixed economy context where there exist both public and private providers for curative care services with an assumption that the government must play the role of regulator and is expected to guarantee health care services in a welfare system. Failure of the government to guarantee health care for its masses is a concern and must be perceived as a failure of public sector provisioning. Public Health programmes have a slightly different mandate to qualify it as one as it must be grounded in public health evidence at a population level targeted at specific public health problems every country is facing. There has been a precedence to follow public health evidence before upscaling any large-scale programme of the entire nation. The case of mitanin to ASHA programme and homebased neonatal care (HBNC) and other maternal health interventions and the old Narangwal Nutrition study for child survival in India are classic 'public health evidences' that was created based on specific population based engagements that has demonstrated results. ${ }^{9}$ Several of the recent flagship programmes like Ayushman Bharat and the recent decision to roll out a vaccination programme all call for public health evidence to qualify it as public health programmes for the nation. It is important to articulate what is the expected population level goal after specific duration of implementing any of these programmes. It is not very clear about the public health goal expected out of these programmes? Was there an evidence of a successful implementation as a pilot intervention? Traditional public health approaches and evidence are very clear on the conditions one should follow to upscale a successful intervention on a larger scale. (i) The interventions should be feasible enough to implement it within a societal context by considering the people's living and working conditions (ii) There must be strong evidence that has demonstrated its success in a smaller population (community) and was able to successfully reduce the problem significantly in that specific community.(iii) There must be ethical justification for implementing this specific intervention as compared to all other possible alternatives currently available so that the benefits are always more than the risk if any accrued through the intervention. ${ }^{10}$

Any improvement in the field of public health in a country will be possible only if it acknowledges and follows these basic principles of the profession. There is a need to generate 'public health evidence', which is distinct from biomedical evidence as in the case of 'vaccine effectiveness' and several other interventions. ${ }^{11}$ There is an important role for pilot studies and innovations to generate public health evidence and must be considered as a pre-requisite for proposing any large-scale national level public health programmes. Most of the national health programmes in India did follow some kind of evidence before upscaling it into country-wide health programmes. The failure to acknowledge the need for public health evidence not only will be a failure to acknowledge the profession and its development in the country but more serious is its failure to create improvement in the population health of its people.

\section{References}

1 Bangdiwala SI, Tucker JD, Zodpey S, et al. Public health education in India and China: history, opportunities, and challenges. Publ. Health Rev. 2011 Jun 1;33(1): 204-224. Sharma A, Zodpey SP. Transforming public health education in India through networking and collaborations: opportunities and challenges. Indian Journal of Public Health. 2013 Jul 1; 57(3): 155.

2 George M. Public health-oriented healthcare systems: need for a modified typology. Natl. Med. J. India. 2019 May 1;32(3):171.

3 Weed DL. Towards a philosophy of public health. J Epidemiol Community. 1999 Feb; 53(2):99.

4 Link BG, Phelan JC. Mckeown and the idea that Social conditions are fundamental causes of disease. Am. J. Publ. Health. 2002;92(5):730-732.

5 Kunitz SJ. Holism and the idea of general susceptibility to disease. Int. J. Epidemiol. 2002 Aug 1;31(4):722-729.

6 Kotwal A, Yadav AK, Yadav J, Kotwal J, Khune S. Predictive models of COVID-19 in India: a rapid review. Med. J. Armed. Forces India. 2020 Oct 1;76(4):377-386.

7 Golechha M. India's Bhilwara COVID-19 containment policy response: lessons for public health policy makers globally. J. of Global Health. 2020 Dec;10(2).

8 WHO Responding to COVID 19- Learnings from Kerala, WHO new, https://www. who.int/india/news/feature-stories/detail/responding-to-covid-19-learnings-fromkerala accessed on 16th December 2020.

9 Lahariya C, Khanna R, Nandan D. Primary health care and child survival in India. Indian J. Pediatr. 2010 Mar;77(3):283-290.

10 Rychetnik L, Frommer M, Hawe P, Shiell A. Criteria for evaluating evidence on public health interventions. J. Epidemiol. Community. 2002 Feb 1;56(2):119-127.

11 Brownson RC, Fielding JE, Maylahn CM. Evidence-based public health: a fundamental concept for public health practice. Annu. Rev. Publ. Health. 2009 Apr 21; 30:175-201. 